\title{
Front Matter: Volume 8088
}

, "Front Matter: Volume 8088," Proc. SPIE 8088, Diffuse Optical Imaging III, 808801 (13 July 2011); doi: 10.1117/12.903806

SPIE. Event: European Conferences on Biomedical Optics, 2011, Munich, Germany 


\title{
PROGRESS IN BIOMEDICAL OPTICS AND IMAGING
}

Vol. 12, No. 42

\section{Diffuse Optical Imaging III}

\author{
Andreas H. Hielscher \\ Paola Taroni \\ Editors
}

22-24 May 2011

Munich, Germany

Sponsored and Published by

SPIE

The Optical Society of America (United States)

Cooperating Organisations

Deutsche Gesellschaft für Lasermedizin (Germany)

Visions for Better Healthcare-Biophotonics Research Program (Germany)

With Support From

Air Force Office of Scientific Research (United States)

Photonics 4 Life-European Network of Excellence for Biophotonics (Germany)

Student Award Sponsors

Toptica Photonics AG (Germany)

ThorLabs (United Kingdom) 
The papers included in this volume were part of the technical conference cited on the cover and title page. Papers were selected and subject to review by the editors and conference program committee. Some conference presentations may not be available for publication. The papers published in these proceedings reflect the work and thoughts of the authors and are published herein as submitted. The publisher is not responsible for the validity of the information or for any outcomes resulting from reliance thereon.

Please use the following format to cite material from this book:

Author(s), "Title of Paper," in Diffuse Optical Imaging III, edited by Andreas H. Hielscher, Paola Taroni, Proceedings of SPIE-OSA Biomedical Optics Vol. 8088 (SPIE, Bellingham, WA, 2011) Article CID Number.

ISSN 1605-7422

ISBN 9780819486851

Published by

SPIE

P.O. Box 10, Bellingham, Washington 98227-0010 USA

Telephone +1 3606763290 (Pacific Time) · Fax +1 3606471445

SPIE.org

Copyright @ 2011, Society of Photo-Optical Instrumentation Engineers.

Copying of material in this book for internal or personal use, or for the internal or personal use of specific clients, beyond the fair use provisions granted by the U.S. Copyright Law is authorized by SPIE subject to payment of copying fees. The Transactional Reporting Service base fee for this volume is $\$ 18.00$ per article (or portion thereof), which should be paid directly to the Copyright Clearance Center (CCC), 222 Rosewood Drive, Danvers, MA 01923. Payment may also be made electronically through CCC Online at copyright.com. Other copying for republication, resale, advertising or promotion, or any form of systematic or multiple reproduction of any material in this book is prohibited except with permission in writing from the publisher. The CCC fee code is 1605 $7422 / 11 / \$ 18.00$.

Printed in the United States of America.

Publication of record for individual papers is online in the SPIE Digital Library.

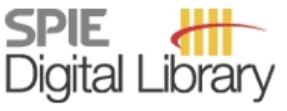

SPIEDigitallibrary.org

Paper Numbering: Proceedings of SPIE follow an e-First publication model, with papers published first online and then in print and on CD-ROM. Papers are published as they are submitted and meet publication criteria. A unique, consistent, permanent citation identifier (CID) number is assigned to each article at the time of the first publication. Utilization of CIDs allows articles to be fully citable as soon as they are published online, and connects the same identifier to all online, print, and electronic versions of the publication. SPIE uses a six-digit CID article numbering system in which:

- The first four digits correspond to the SPIE volume number.

- The last two digits indicate publication order within the volume using a Base 36 numbering system employing both numerals and letters. These two-number sets start with 00, 01, 02, 03, 04, $05,06,07,08,09,0 A, 0 B \ldots$. OZ, followed by 10-1Z, 20-2Z, etc.

The CID number appears on each page of the manuscript. The complete citation is used on the first page, and an abbreviated version on subsequent pages. Numbers in the index correspond to the last two digits of the six-digit CID number. 


\section{Contents}

xi Conference Committee

SESSION 1 MAPPING OF BRAIN FUNCTION WITH DOI AND MULTIMODALITY IMAGING

808802 Assessment of cortical response during motor task in adults by a multimodality approach based on fNIRS-EEG, fMRI-EEG, and TMS (Invited Paper) [8088-01]

A. Torricelli, D. Contini, M. Caffini, L. Zucchelli, R. Cubeddu, Politecnico di Milano (Italy); L. Spinelli, Istituto di Fotonica e Nanotecnologie, CNR (Italy); E. Molteni, A. M. Bianchi, G. Baselli, S. Cerutti, Politecnico di Milano (Italy); E. Visani, I. Gilioli, D. Rossi Sebastiano, E. Schiaffi, F. Panzica, S. Franceschetti, Fondazione IRCCS Istituto Neurologico Carlo Besta (Italy)

808803 Simultaneous measurement of time-domain fNIRS and physiological signals during a cognitive task [8088-03]

A. Jelzow, Physikalisch-Technische Bundesanstalt (Germany); I. Tachtsidis, Univ. College London (United Kingdom); E. Kirilina, M. Niessing, Dahlem Institute of Neuroimaging of Emotion (Germany) and Free Univ. of Berlin (Germany); R. Brühl, H. Wabnitz,

Physikalisch-Technische Bundesanstalt (Germany); A. Heine, Free Univ. of Berlin (Germany);

B. Ittermann, R. Macdonald, Physikalisch-Technische Bundesanstalt (Germany)

808806 Slow spontaneous hemodynamic oscillations during sleep measured with near-infrared spectroscopy [8088-05]

J. Virtanen, T. Näsi, Aalto Univ. (Finland) and Helsinki Univ. Central Hospital (Finland);

T. Noponen, Turku Univ. Hospital (Finland); J. Toppila, T. Salmi, Helsinki Univ. Central Hospital

(Finland); R. J. Ilmoniemi, Aalto Univ. (Finland) and Helsinki Univ. Central Hospital (Finland)

\section{SESSION 2 NEW METHODS AND TECHNIQUES FOR BRAIN IMAGING}

808807 Robot-assisted motor activation monitored by time-domain optical brain imaging [8088-06] O. Steinkellner, H. Wabnitz, Physikalisch-Technische Bundesanstalt (Germany); S. Schmid, R. Steingräber, Technische Univ. Berlin (Germany); H. Schmidt, J. Krüger, Fraunhofer-Institu† für Produktionsanlagen und Konstruktionstechnik (Germany); R. Macdonald, Physikalisch-Technische Bundesanstalt (Germany)

808808 Simultaneous imaging of haemoglobin oxygenation and blood flow with RGB reflectometry and LASCA during stroke in rats [8088-07]

A. Steimers, Univ. of Applied Sciences Koblenz (Germany); M. Gramer, M. Takagaki, R. Graf, Max Planck Institute for Neurological Research (Germany); M. Kohl-Bareis, Univ. of Applied Sciences Koblenz (Germany)

808809 Anatomical brain atlas for NIRS measurements of brain activation [8088-08] M. Caffini, L. Zucchelli, D. Contini, R. Cubeddu, Politecnico di Milano (Italy); L. Spinelli, Istituto di Fotonica e Nanotecnologie, CNR (Italy); D. Boas, Martinos Ctr. for Biomedical Imaging (United States); A. Torricelli, Politecnico di Milano (Italy) 
8088 OA Non-contact time-domain scanning brain imager: results of proof of principle tests [8088-09] M. Mazurenka, A. Jelzow, B. Ebert, H. Wabnitz, Physikalisch-Technische Bundesanstalt (Germany); D. Contini, ITT, Politecnico di Milano (Italy); L. Spinelli, Istituto di Fotonica e Nanotecnologie, CNR (Italy); A. Pifferi, ITT, Politecnico di Milano (Italy) and Istituto di Fotonica e Nanotecnologie, CNR (Italy); A. Dalla Mora, ITT, Politecnico di Milano (Italy); A. Tosi, Politecnico di Milano (Italy); R. Macdonald, Physikalisch-Technische Bundesanstalt (Germany)

8088 OC Time-resolved and spectral-resolved optical imaging to study brain hemodynamics in songbirds [8088-11]

S. Mottin, Lab. Hubert Curien, CNRS, Univ. Jean Monnet Saint-Etienne (France); B. Montcel, CREATIS, CNRS, Univ. Lyon 1 (France); H. Guillet de Chatellus, Lab. de spectrométrie physique, CNRS, Univ. de Grenoble (France); S. Ramstein, Lab. Hubert Curien, CNRS, Univ. de Lyon (France); C. Vignal, N. Mathevon, Ctr. de Neurosciences Paris-Sud, CNRS, Univ. Jean Monnet Saint-Etienne (France)

8088 OE Comparison of temporal response to cerebral blood flow measured by laser speckle flowgraphy and laser Doppler flowmetry [8088-13]

M. Kusano, H. Nakayama, Keio Univ. (Japan); H. Takuwa, K. Masamoto, I. Kanno, National Institute of Radiological Sciences (Japan); E. Okada, Keio Univ. (Japan)

\section{SESSION 3 HUMAN HEAD MODELS}

$8088 \mathrm{OH} \quad$ Non-invasive determination of the optical properties of the human head using a neural network [8088-16]

M. Jäger, A. Kienle, Institut für Lasertechnologien in der Medizin und Meßtechnik (Germany)

8088 Ol Three-dimensional superposition of diffuse optical tomography results and subjacent anatomic structures [8088-17]

C. Habermehl, Berlin Neurolmaging Ctr. (Germany); C. Schmitz, Berlin Neurolmaging Ctr. (Germany) and NIRx Medizintechnik GmbH (Germany); J. Mehnert, S. Holtze, Berlin Neurolmaging Ctr. (Germany) and Max Planck Institute for Human Cognitive and Brain Sciences (Germany); J. Steinbrink, Berlin Neurolmaging Ctr. (Germany) and Charité Universitätsmedizin (Germany)

$8088 \mathrm{JJ}$ Segmentation of magnetic resonance images to construct human head model for diffuse optical imaging [8088-18]

K. Kurihara, Keio Univ. (Japan); H. Kawaguchi, National Institute of Radiological Sciences (Japan); Y. Takahashi, Keio Univ. (Japan); T. Obata, National Institute of Radiological

Sciences (Japan); E. Okada, Keio Univ. (Japan)

\section{SESSION 4 MODELING OF LIGHT PROPAGATION}

8088 OK Simulating light propagation: towards realistic tissue models [8088-19]

H. G. Akarçay, J. Rička, Univ. of Bern (Switzerland)

$8088 \mathrm{OL}$ Analytical solutions of the radiative transfer equation for the fluence and radiance in infinite turbid media [8088-20]

A. Liemert, A. Kienle, Institut für Lasertechnologien in der Medizin und Meßtechnik (Germany) 
808800 Importance of considering exact boundary conditions of the probe geometry for determination of the optical properties of turbid media [8088-23]

O. Fugger, A. Kienle, Institut für Lasertechnologien in der Medizin und Meßtechnik (Germany)

8088 OP Spectral reflectance fitting based on Monte Carlo simulation using a multi-layered skin tissue model [8088-24]

Y. Aizu, Muroran Institute of Technology (Japan); T. Maeda, Kushiro National College of Technology (Japan); T. Kuwahara, T. Hirao, Shiseido Research Ctr. (Japan)

\section{SESSION 5 OPTICAL TOMOGRAPHY I}

8088 OR Optical projection tomography for light scattering media [8088-26]

V. Y. Soloviev, S. R. Arridge, Univ. College London (United Kingdom)

8088 OS Diffuse optical tomography of absorption in biological media using time-dependent parabolic simplified spherical polynomials equations [8088-27]

J. Bouza Domínguez, Y. Bérubé-Lauzière, Univ. de Sherbrooke (Canada)

8088 OT A semi-analytical perturbation model for diffusion tomogram reconstruction from time-resolved optical projections [8088-28]

A. B. Konovalov, V. V. Vlasov, A. S. Uglov, Russian Federal Nuclear Ctr., Zababakhin Institute of Applied Physics (Russian Federation); V. V. Lyubimov, S.I. Vavilov State Optical Institute Corp. (Russian Federation)

\section{SESSION 6 OPTICAL TOMOGRAPHY II}

$8088 \mathrm{OV}$ Hyperspectral fluorescence tomography of quantum dots using the simplified spherical harmonics equations [8088-30]

A. D. Klose, Y. Tekabe, L. Johnson, Columbia Univ. (United States)

8088 OW Toward absolute quantification in CW-FDOT systems: use of a priori information [8088-31] A. Planat-Chrétien, A. Koenig, J.-G. Coutard, L. Hervé, M. Brambilla, J.-M. Dinten, CEA/LETI (France)

8088 0X Development of image reconstruction algorithms for fluorescence diffuse optical tomography using total light approach [8088-32]

S. Okawa, The Univ. of Electro-Communications (Japan); H. Yamamoto, FUJIFILM Corp. (Japan); Y. Miwa, Univ. of Tsukuba (Japan); Y. Yamada, The Univ. of Electro-Communications (Japan)

8088 OY Multi-wavelength diffusive optical tomography using independent component analysis and time reversal algorithms [8088-33]

M. Alrubaiee, B. Wu, The City College of New York (United States) and Graduate Ctr., City Univ. of New York (United States); M. Xu, Fairfield Univ. (United States); W. Cai, The City College of New York (United States) and Graduate Ctr., City Univ. of New York (United States); J. A. Koutcher, Memorial Sloan-Kettering Cancer Ctr. (United States); S. K. Gayen, The City College of New York (United States) and Graduate Ctr., City Univ. of New York (United States) 
808811 3D near-infrared imaging based on a single-photon avalanche diode array sensor (Invited Paper) [8088-37]

J. Mata Pavia, Univ. Hospital Zürich (Switzerland) and Ecole Polytechnique Fédérale de Lausanne (Switzerland); E. Charbon, Ecole Polytechnique Fédérale de Lausanne (Switzerland) and Technische Univ. Delft (Netherlands); M. Wolf, Univ. Hospital Zürich (Switzerland)

\section{SESSION 8 NOVEL INSTRUMENTATION}

808814 Quantitative photoacoustic blood oxygenation measurement of whole porcine blood samples using a multi-wavelength semiconductor laser system [8088-40]

C.-S. Friedrich, M. P. Mienkina, C. Brenner, N. C. Gerhardt, Ruhr-Univ. Bochum (Germany);

M. Jörger, A. Strauß, llias-medical GmbH (Germany); M. F. Beckmann, G. Schmitz,

M. R. Hofmann, Ruhr-Univ. Bochum (Germany)

\section{SESSION 9 EXPERIMENTAL METHODS}

808817 Time-domain diffuse optical spectroscopy beyond $1100 \mathrm{~nm}$ : initial feasibility study [8088-43] A. Pifferi, Politecnico di Milano (Italy) and Istituto di Fotonica e Nanotecnologie, CNR (Italy); I. Bargigia, Politecnico di Milano (Italy); P. Taroni, Politecnico di Milano (Italy) and Istituto di Fotonica e Nanotecnologie, CNR (Italy); A. Farina, Politecnico di Milano (Italy); R. Cubeddu, Politecnico di Milano (Italy) and Istituto di Fotonica e Nanotecnologie, CNR (Italy)

808818 SNR enhancement by using polarized light for cortex functional imaging [8088-44] A. Da Silva, Institut Fresnel, CNRS, Aix-Marseille Univ. (France); P. Stahl, Institut Fresnel, CNRS, Aix-Marseille Univ. (France) and Institut de Neurosciences Cognitives de la Méditerranée, CNRS (France); C. Deumié, Institut Fresnel, CNRS, Aix-Marseille Univ. (France); I. Vanzetta, Institut de Neurosciences Cognitives de la Méditerranée, CNRS (France)

80881 A Analysis of the diffuse reflectance spectra of skin due to detection system [8088-47] S. Takano, E. Okada, Keio Univ. (Japan)

8088 1B Evaluation of the position resolution of NIR topography by localised visual stimulation [8088-48]

H. Kakuta, E. Okada, Keio Univ. (Japan)

8088 1C Simple method of improving sensitivity for diffuse reflective optical tomography: simulation and a phantom study [8088-49]

K. Fukuda, K. Koishi, T. Murayama, Tokyo Metropolitan College of Industrial Technology (Japan)

8088 ID Phantom experiments for quantitative evaluation of topographic image by mapping algorithm [8088-50]

Y. Yoshida, H. Kakuta, Keio Univ. (Japan); H. Kawaguchi, National Institute of Radiological Sciences (Japan); E. Okada, Keio Univ. (Japan) 
$80881 \mathrm{~B} \quad$ Breast density assessment by means of time domain optical mammography at 635-1060 nm [8088-51]

P. Taroni, A. Pifferi, G. Quarto, Politecnico di Milano (Italy); L. Spinelli, Istituto di Fotonica e Nanotecnologie, CNR (Italy); A. Torricelli, R. Cubeddu, Politecnico di Milano (Italy);

F. Abbate, A. Villa, N. Balestreri, S. Menna, E. Cassano, European Institute of Oncology (Italy)

$80881 \mathrm{G}$ Oxygenation of the calf muscle during an incremental, intermittent walking exercise assessed by NIRS [8088-53]

S. Härtel, C. Kutzner, Karlsruher Institute of Technology (Germany); D. Schneider, S. Grieger, Univ. of Applied Sciences Koblenz (Germany); M. Neumaier, Karlsruher Institute of

Technology (Germany); M. Kohl-Bareis, Univ. of Applied Sciences Koblenz (Germany)

\section{POSTER SESSION}

808811 Comparison of polarized light penetration depth in scattering media [8088-46]

S. Rehn, Institut Fresnel, CNRS, Aix-Marseille Univ. (France) and CEA, LETI (France);

A. Planat-Chrétien, M. Berger, J.-M. Dinten, CEA, LETI (France); C. Deumié, A. da Silva, Institut Fresnel, CNRS, Aix-Marseille Univ. (France)

$80881 \mathrm{~J}$ Depth-resolved quantitative measurement of cerebral blood flow using broad-band near infrared spectroscopy and a two-layer head model [8088-55]

V. Toronov, Ryerson Univ. (Canada); J. Elliott, Lawson Health Research Institute (Canada);

T.-Y. Lee, Lawson Health Research Institute (Canada) and Robarts Research Institute (Canada); K. St. Lawrence, Lawson Health Research Institute (Canada)

8088 1K Development of a multi-view multi-spectral bioluminescence tomography small animal imaging system [8088-56]

J. A. Guggenheim, H. Dehghani, H. Basevi, I. B. Styles, J. Frampton, The Univ. of Birmingham (United Kingdom)

$80881 \mathrm{M}$ Principal and independent component analysis of concomitant functional near infrared spectroscopy and magnetic resonance imaging data [8088-58]

I. Schelkanova, V. Toronov, Ryerson Univ. (Canada)

8088 iN Determination of the reduced scattering and absorption coefficients by spatially resolved reflectance and total reflectance measurements [8088-59]

F. Foschum, M.-T. Heine, A. Kienle, Institut für Lasertechnologien in der Medizin und Meßtechnik (Germany)

808810 Frequency domain diffuse fluorescence tomography for detection of deep lesions [8088-60] U. J. Netz, I. Gersonde, J. Toelsner, G. Illing, Laser- und Medizin-Technologie Berlin (Germany)

8088 IP Uncertainty analysis for fluorescence tomography with Monte Carlo method [8088-62] A. Reinbacher-Köstinger, M. Freiberger, H. Scharfetter, Graz Univ. of Technology (Austria) 
$80881 Q$ Using the topological derivative for initializing a Markov-chain Monte Carlo reconstruction in fluorescence tomography [8088-63]

M. Freiberger, Graz Univ. of Technology (Austria); A. Laurain, Univ. of Graz (Austria);

M. Hintermüller, Humboldt-Univ. zu Berlin (Germany); A. Köstinger, H. Scharfetter, Graz Univ.

of Technology (Austria)

8088 IR Numerical modeling of light propagation in biological tissues: time-resolved 3D simulations based on light diffusion model and FDTD solution of Maxwell's equations [8088-64]

N. Ortega-Quijano, Univ. of Cantabria (Spain); O. G. Romanov, Belarusian State Univ. (Belarus); F. Fanjul-Vélez, I. Salas-García, Univ. of Cantabria (Spain); A. L. Tolstik, Belarusian State Univ. (Belarus); J. L. Arce-Diego, Univ. of Cantabria (Spain)

8088 IT BEM-NIRFAST: open source software for 3D image-guided near infrared spectroscopy using boundary element method [8088-66]

S. Srinivasan, H. R. Ghadyani, M. Jeremyn, Dartmouth College (United States)

$80881 \mathrm{U}$ Three-dimensional image-guided fluorescence using boundary element method and dual reciprocity method [8088-67]

R. Yang, S. Srinivasan, R. L. S. Drysdale, Dartmouth College (United States)

8088 IV 3D-surface reconstruction method for diffuse optical tomography phantoms and tissues using structured and polarized light [8088-68]

K. Baum, R. Hartmann, T. Bischoff, F. Himmelreich, J. T. Heverhagen, Philipps Univ. Marburg (Germany)

Author Index 


\title{
Conference Committee
}

\author{
General Chairs
}

Christoph K. Hizenberger, Medizinische Universität Wien (Austria)

Brian W. Pogue, Dartmouth College (United States)

Programme Chairs

Peter E. Andersen, Technical University of Denmark (Denmark)

Irene Georgakoudi, Tufts University (United States)

Conference Chairs

Andreas H. Hielscher, Columbia University (United States)

Paola Taroni, Politecnico di Milano (Italy)

\section{Conference Committee}

Hamid Dehghani, The University of Birmingham (United Kingdom)

Turgut Durduran, ICFO - Instituto de Ciencias Fotónicas (Spain)

Xavier Intes, Rensselaer Polytechnic Institute (United States)

Adam Liebert, Institute of Biocybernetics and Biomedical Engineering (Poland)

Subhadra Srinivasan, Dartmouth College (United States)

Heidrun Wabnitz, Physikalisch-Technische Bundesanstalt (Germany)

Martin Wolf, University Hospital Zürich (Switzerland)

Yukio Yamada, The University of Electro-Communications (Japan)

\section{Session Chairs}

1 Mapping of Brain Function with DOI and Multimodality Imaging Heidrun Wabnitz, Physikalisch-Technische Bundesanstalt (Germany) Eiji Okada, Keio University (Japan)

2 New Methods and Techniques for Brain Imaging

Eiji Okada, Keio University (Japan)

Martin Wolf, University Hospital Zürich (Switzerland)

3 Human Head Models

Martin Wolf, University Hospital Zürich (Switzerland)

Hamid Dehghani, The University of Birmingham (United Kingdom) 
$4 \quad$ Modeling of Light Propagation

Hamid Dehghani, The University of Birmingham (United Kingdom)

5 Optical Tomography I

Hamid Dehghani, The University of Birmingham (United Kingdom)

6 Optical Tomography II

Hamid Dehghani, The University of Birmingham (United Kingdom)

7 Optical Tomography III

Paola Taroni, Politecnico di Milano (Italy)

8 Novel Instrumentation

Turgut Durduran, ICFO - Instituto de Ciencias Fotónicas (Spain)

9 Experimental Methods

Paola Taroni, Politecnico di Milano (Italy)

10 Clinical Applications

Adam Liebert, Institute of Biocybernetics and Biomedical Engineering (Poland)

Turgut Durduran, ICFO - Instituto de Ciencias Fotónicas (Spain)

Poster Session

Paola Taroni, Politecnico di Milano (Italy) 